\title{
Quantum state of wormholes and path integral
}

\author{
Luis J. Garay \\ Instituto de Optica, Consejo Superior de Investigaciones Científicas, Serrano 121, E-28006 Madrid, Spain
}

(Received 28 March 1991)

\begin{abstract}
The quantum state of a wormhole can be represented by a path integral over all asymptotically Euclidean four-geometries and all matter fields which have prescribed values, the arguments of the wave function, on a three-surface $S$ which divides the spacetime manifold into two disconnected parts. The ground-state wave function is picked out by requiring that there be no matter excitations in the asymptotic region. Once the path integrals over the lapse and shift functions are evaluated, the requirement that the spacetime be asymptotically Euclidean can be accomplished by fixing the asymptotic gravitational momentum in the remaining path integral. It is claimed that no wave function exists which corresponds to asymptotic field configurations such that the effective gravitational constant is negative in the asymptotic region. The wormhole wave functions are worked out in minisuperspace models with massless minimal and conformal scalar fields.
\end{abstract}

\section{INTRODUCTION}

Wormholes have been considered as instantons, solutions of the Euclidean Einstein equations, which consist of two asymptotically Euclidean regions connected by a throat [1-7]. These classical wormholes are saddle points of the Euclidean action and, therefore, allow the Euclidean path integral to be approximated semiclassically. One makes the dilute wormhole approximation in which the wormhole ends are far apart from each other so that one can consider that wormholes do not interact and then can be treated separately. Wormholes on the Planck scale may affect the constants of nature and, in particular, may provide a mechanism for the vanishing of the cosmological constant [8-10]. Wormholes may play an important role in solving problems associated with the complete evaporation and disappearance of black holes [2]. However, classical wormholes may only exist for very special types of matter, those which allow the Ricci tensor to have negative eigenvalues $[1,11,12]$. This may place a strong restriction on the possibility that wormholes have any role in these processes.

However, we need not restrict ourselves to such a semiclassical treatment and the special types of matter that it requires. More generally, one can regard wormholes as solutions of the quantum Wheeler-DeWitt equation with some suitable boundary conditions. Hawking and Page [13] have proposed that the boundary conditions should reflect the fact that the four-geometry is nonsingular, even when the three-geometry degenerates, i.e., the wave function should be regular in some suitable way when the three-geometry collapses to zero; also, the wave function should be exponentially damped for large threegeometries owing to considering asymptotically Euclidean four-geometries.

I shall concentrate on the behavior of spacetime and matter fields when the three-geometry is large. In the minisuperspace models that I work out in this paper, the first boundary condition is automatically satisfied, the wave functions are regular when the three-geometry collapses. However, these regular solutions can be written as linear combinations of solutions that do not close off with a four-geometry and, hence, have a nonzero flux of a conserved quantity across any three-surface.

In this paper, I shall assume that the spacetime manifold is divided into two disconnected parts by the threesurface $S$ in which the arguments of the wave function are defined (see, however [14]).

The wormhole wave function can be represented as the path integral over all asymptotically Euclidean fourgeometries that match a given compact three-geometry which is the argument of the wave function. This can be interpreted as saying that there are no gravitational excitations at infinity (i.e., at large distance compared with the characteristic scale of the wormhole). An extra surface term which eliminates the infinite contribution that comes from the asymptotically Euclidean region should be added to the action. It will be seen that, once the path integrals over the lapse and shift functions have been evaluated, the requirement that the spacetime is asymptotically Euclidean is equivalent to the fixation of the asymptotic gravitational momentum.

The ground state will be picked by restricting the class of matter fields over which one also integrates. It will consist of all regular matter fields which have no sources in the asymptotic region. This reflects the fact that there are no matter excitations at infinity. This is accomplished by requiring that the matter Hamiltonian must vanish asymptotically. If one considers a coupled scalar field with a potential as the matter content, this means that, at infinity, the field must approach a homogeneous configuration at which the potential has a vanishing minimum. When the scalar field potential is identically zero, any asymptotic homogeneous field configuration will correspond to a situation in which there are no matter excitations, i.e., to a ground state, rather than to an excited state [15]. Therefore, there will be an infinite number of ground states. The excited wormhole wave 
functions will be represented as path integrals over the same kind of four-geometries but the matter fields will have sources at infinity. This can be interpreted as saying that there are particles passing through the wormhole.

Section II is a review of the canonical formalism of general relativity in which a coupled scalar field with a potential is considered for the matter content. Also, an action which includes the appropriate surface term for asymptotically Euclidean spacetimes is given. The gauge-fixing conditions are discussed in Sec. III. In Sec. IV, the path integrals over the lapse and shift functions are evaluated and the class of three-metrics and matter fields over which one has to integrate in order to obtain the ground-state wave function for wormholes is defined precisely. Section $\mathrm{V}$ is devoted to minisuperspace models in which the matter content is a massless scalar field minimally coupled to Friedmann-Robertson-Walker (FRW) and Kantowski-Sachs spacetimes. In Sec. VI, the wormhole wave functions for the minisuperspace that consists of a FRW spacetime and a conformally coupled scalar field are found. I summarize and conclude in Sec. VII.

\section{CLASSICAL ACTION AND CANONICAL FORMALISM}

The study of wormholes in the dilute wormhole approximation requires a four-manifold which is asymptotically Euclidean. The metric can be written in the form

$d s^{2}=\left(N^{2}+N^{i} N_{i}\right) d \tau^{2}+2 N_{i} d \tau d x^{i}+g_{i k} d x^{i} d x^{k}$,

where $N$ is the lapse function which measures the proper time separation of three-surfaces of constant $\tau, N^{i}$ are the shift functions which measure the deviation of the lines of constant $x^{i}$ from the normal direction to the threesurfaces and $g_{i k}$ is the metric in the three-surfaces $\tau=$ const, which will be chosen to be compact. Each of these sections will divide the spacetime manifold into two disconnected parts. In particular, the three-surface which will be the argument of the wave function will correspond to the section $\tau=0$. For the matter content, I shall choose a scalar field, which will be coupled to this spacetime, with a potential $V(\Phi)$.

The Euclidean action will be

$$
\begin{aligned}
\widetilde{I}= & \int_{0}^{\infty} d \tau \int d^{3} x N g^{1 / 2}\left[-\frac{m_{P}^{2}}{16 \pi}\left[1-\frac{8 \pi}{m_{P}^{2}} \xi \Phi^{2}\right]{ }^{(4)} R+\frac{1}{2} \frac{1}{N^{2}} \dot{\Phi}^{2}-\frac{N^{i}}{N^{2}} \dot{\Phi} \nabla_{i} \Phi+\frac{1}{2}\left[g^{i k}+\frac{N^{i} N^{k}}{N^{2}}\right] \nabla_{i} \Phi \nabla_{k} \Phi+V(\Phi)\right] \\
& -\frac{m_{P}^{2}}{8 \pi} \int d^{3} x g^{1 / 2} K\left[1-\frac{8 \pi}{m_{P}^{2}} \xi \Phi^{2}\right]
\end{aligned}
$$

where the overdot denotes a partial derivative with respect to $\tau, g=\operatorname{det}_{i k}$ and $K$ is the trace of the second fundamental form of the three-surface:

$$
K_{i k}=\frac{1}{2 N}\left(\dot{g}_{i k}+2 N_{(i \mid k)}\right) \text {. }
$$

The coupling ${ }^{(4)} R \xi \Phi^{2}$, where $\xi$ is an arbitrary numerical factor, is the only possible local scalar coupling of this sort which has the correct dimensions.

For the sake of simplicity, we shall perform the calculations for the case $\xi=0$, which corresponds to the minimal coupling. The extension to the conformal coupling $\xi=1 / 6$ is straightforward. However, for other couplings, which will not be considered here, the system is degenerate in the sense that one cannot globally invert the relations between the momenta and the derivatives of the canonical variables [15a].

In the canonical formulation of general relativity, the components of the three-metric $g_{i k}$ and the matter field $\Phi$ are regarded as the canonical coordinates whose canonical conjugate momenta are

$$
\pi^{i k}=\frac{\delta \widetilde{I}}{\delta \dot{g}_{i k}}=\frac{m_{P}^{2}}{16 \pi} g^{1 / 2}\left(K^{i k}-g^{i k} K\right)
$$

and

$$
\pi_{\Phi}=\frac{\delta \widetilde{I}}{\delta \dot{\Phi}}=N^{-1} g^{1 / 2}\left(\dot{\Phi}-N^{i} \nabla_{i} \Phi\right)
$$

Then, the Euclidean action can be written

$$
\widetilde{I}=\int_{0}^{\infty} d \tau \int d^{3} x\left(\pi^{i k} \dot{g}_{i k}+\pi_{\Phi} \dot{\Phi}-N \mathscr{H}-N^{i} \mathscr{H}_{i}\right),
$$

in which $\mathcal{H}$ and $\mathscr{H}_{i}$ are the Hamiltonian generators

$$
\begin{aligned}
\mathscr{H}= & \frac{16 \pi}{m_{P}^{2}} G_{i j k l} \pi^{i j} \pi^{k l}+\frac{m_{P}^{2}}{16 \pi} g^{1 / 2} R \\
& +\frac{1}{2} g^{-1 / 2} \pi_{\Phi}^{2}-\frac{1}{2} g^{1 / 2} g^{i k} \nabla_{i} \Phi \nabla_{k} \Phi-g^{1 / 2} V(\phi), \\
\mathscr{H}_{i}= & -2 \nabla_{k} \pi_{i}^{k}-\pi_{\Phi} \nabla_{i} \Phi,
\end{aligned}
$$

where

$$
G_{i j k l}=\frac{1}{2} g^{-1 / 2}\left(g_{i k} g_{j l}+g_{i l} g_{j k}-g_{i j} g_{k l}\right)
$$

is the metric on superspace and $R$ is the curvature associated with the metric $g_{i k}$ in the three-surface.

An additional term must be introduced into the action (2.6) when one requires that the spacetime is asymptotically Euclidean. This term is

$$
\frac{m_{P}^{2}}{8 \pi} \int d^{3} x g^{1 / 2} K_{0}
$$

where $K_{0}$ is the trace of the second fundamental form of the asymptotic three-surface when it is embedded in Eu- 
clidean spacetime [16]. From the expression for the gravitational momentum (2.4), one can easily see that this surface term can be written in terms of $\pi^{i k}$ and $g_{i k}$ as

$$
-\left.\int d^{3} x \pi^{i k} g_{i k}\right|_{\tau=\infty}
$$

This term makes the action of Euclidean spacetime vanish and, therefore, renders the action of asymptotically Euclidean spacetime finite. It removes the infinite contribution that comes from the asymptotic region. Then, the correct form of the action that takes into account the fact that the spacetime is asymptotically Euclidean is

$$
I=\widetilde{I}-\left.\int d^{3} x \pi^{i k} g_{i k}\right|_{\tau=\infty},
$$

where $\widetilde{I}$ is given by (2.6).

Variations of the action with respect to the threemetric, the matter field, and their respective conjugate momenta yield the classical equations of motion,

$$
\begin{aligned}
& \dot{g}_{i k}=\left\{g_{i k}, H\right\}, \quad \dot{\pi}^{i k}=\left\{\pi^{i k}, H\right\}, \\
& \dot{\Phi}=\{\Phi, H\}, \quad \dot{\pi}_{\Phi}=\left\{\pi_{\Phi}, H\right\},
\end{aligned}
$$

where

$$
H=\int d^{3} x\left(N \mathscr{H}+N^{i} \mathscr{H}_{i}\right)
$$

and variations of the action with respect to the lapse and shift functions yield the constraints

$$
\mathscr{H}=0, \mathscr{H}_{i}=0 \text {. }
$$

The Poisson-brackets algebra of the Hamiltonian constraints $\mathscr{H}_{\mu}=\left(\mathscr{H}, \mathscr{H}_{i}\right)$ for pure gravity $[17,18]$ is not affected by the introduction of the scalar field, as can be easily checked by direct calculation. This algebra is

$$
\left\{\mathscr{H}_{\mu}, \mathscr{H}_{v^{\prime}}\right\}=\kappa_{\mu v^{\prime \prime}}^{\rho^{\prime \prime}} \mathscr{H}_{\rho^{\prime \prime}},
$$

where the Einstein summation convention applies not only to the discrete Greek indices, but also to the continuous space coordinates (specified by primes, double primes or nothing attached to the discrete indices). The only nonvanishing structure functions are

$$
\begin{aligned}
\kappa_{00^{\prime}}^{i^{\prime \prime}} & =-\kappa_{0^{\prime} 0}^{i^{\prime \prime}} \\
& =-g^{i j}\left(x^{\prime \prime}\right) \delta_{, j}\left(x, x^{\prime}\right)\left[\delta\left(x, x^{\prime \prime}\right)+\delta\left(x^{\prime}, x^{\prime \prime}\right)\right] \\
\kappa_{i 0^{\prime}}^{0^{\prime \prime}} & =-\kappa_{0^{\prime} i}^{0^{\prime \prime}}=\delta_{, i}\left(x, x^{\prime}\right)\left[\delta\left(x, x^{\prime \prime}\right)+\delta\left(x^{\prime}, x^{\prime \prime}\right)\right] \\
\kappa_{i j^{\prime}}^{k^{\prime \prime}} & =-\kappa_{j^{\prime} i}^{k^{\prime \prime}}=\delta_{i}^{k} \delta_{, j}\left(x, x^{\prime}\right) \delta\left(x^{\prime}, x^{\prime \prime}\right)+\delta_{j}^{k} \delta_{, i}\left(x, x^{\prime}\right) \delta\left(x, x^{\prime \prime}\right) .
\end{aligned}
$$

The algebra (2.16) will be used in the next section for calculating the variation of the action under general gauge transformations.

\section{GAUGE FIXATION}

The action (2.12) is invariant under certain kinds of gauge transformations. Therefore, when one performs the path integration over four-geometries, the gauge freedom that one has in the action must be removed [17]. For this purpose, consider a general gauge transformation, i.e., an arbitrary change of spacetime coordinates,

$$
\begin{aligned}
& \delta g_{i k}=\left\{g_{i k}, H\left[\epsilon^{\mu}\right]\right\}, \quad \delta \pi^{i k}=\left\{\pi^{i k}, H\left[\epsilon^{\mu}\right]\right\}, \\
& \delta \Phi=\left\{\Phi, H\left[\epsilon^{\mu}\right]\right\}, \delta \pi_{\Phi}=\left\{\pi_{\Phi}, H\left[\epsilon^{\mu}\right]\right\}, \\
& \delta N^{\mu^{\prime \prime}}=\dot{\epsilon}^{\mu^{\prime \prime}}-\kappa_{v \sigma^{\prime}}^{\mu^{\prime \prime}} N^{v} \epsilon^{\sigma^{\prime}},
\end{aligned}
$$

where $N^{\mu}=\left(N, N^{i}\right)$ and $H\left[\epsilon^{\mu}\right]$ is a linear combination of the Hamiltonian constraints with arbitrary coefficients $\epsilon^{\mu}(x, \tau)$ that parametrize the gauge transformation

$$
H\left[\epsilon^{\mu}\right]=\int d^{3} x\left(\epsilon \mathscr{H}+\epsilon^{i} \mathscr{H}_{i}\right) .
$$

Then, the variation of the action (2.12) can be found to be

$$
\begin{gathered}
\delta I=-\int d^{3} x\left\{\epsilon(x, 0)\left[\pi^{i k}\left\{g_{i k}, \mathscr{H}\right\}+\pi_{\Phi}\{\Phi, \mathscr{H}\}-\mathscr{H}\right]_{\tau=0}\right. \\
\left.+\left.\epsilon(x, \infty)\left(\frac{1}{2} \mathscr{H}-g^{1 / 2} V\right)\right|_{\tau=\infty}\right\} .
\end{gathered}
$$

The requirement that the spacetime be asymptotically Euclidean makes the gravitational part of the Hamiltonian (2.7) vanish asymptotically. The boundary condition that picks the ground-state wave function for the wormhole requires that there be no matter excitations in the asymptotically Euclidean region and, therefore, the part of the Hamiltonian which corresponds to the matter content must vanish at infinity. Also as is shown in the next section, these boundary conditions can be satisfied only if the potential has a vanishing minimum in the asymptotic region. Then, it follows that the last term in (3.3) must vanish for the ground state because of the boundary conditions. Therefore, the action (2.12) will be invariant under the gauge transformation (3.1) provided

$$
\epsilon(x, 0)=0 \text {. }
$$

When one performs the path integration, one has to sum over four-geometries, over the representants of the classes of equivalence of four-metrics, each class being composed of all four-metrics which are related by a gauge transformation of the type (3.1) with the boundary condition (3.4). The additional condition

$$
\epsilon^{i}(x, 0)=0
$$

must be also imposed because the argument of the wave function is a three-geometry and therefore, it must be invariant under reparametrizations on the three-surface $\tau=0$. The condition (3.5) fixes the three-metric at $\tau=0$, and, thus, when one removes the gauge freedom in order to choose the four-metric that represents each fourgeometry, this condition allows us to preserve the gauge freedom that should be kept by the wave function.

The gauge-fixing condition that chooses the fourmetrics which will represent all four-geometries in the path integral must be admissible [19], i.e., it must be possible to deform any history into another satisfying the 
gauge condition by a unique gauge transformation; the uniqueness guarantees that the gauge has been fixed completely. The simplest admissible gauge fixation is

$$
N^{(0)}(x, \tau)=1, \quad N^{(0) i}(x, \tau)=0
$$

That this gauge condition is an admissible one, i.e., that for each $N^{\mu}$ there exists a unique gauge transformation which relates it to (3.6), is easy to prove. Let $N^{\mu}(x, \tau)$ be any history. The differential equations in $\epsilon^{\mu}$,

$$
\delta N^{\mu}=N^{\mu}-N^{(0) \mu}=\dot{\epsilon}^{\mu}-\kappa_{v^{\prime} \sigma^{\prime \prime}}^{\mu} N^{(0) v^{\prime}} \epsilon^{\sigma^{\prime \prime}},
$$

must have a unique solution that satisfies the conditions (3.4) and (3.5). Then it will be the unique gauge transformation that deforms $N^{\mu}$ into $N^{(0) \mu}$. These are four linear differential equations of first order and, therefore, the general solution will depend on four arbitrary functions of the spatial coordinates which are uniquely determined by the four conditions (3.4) and (3.5).

\section{PATH INTEGRAL}

Now, the path integral which defines the ground-state wormhole wave function can be written explicitly. It will be given by the sum of $e^{-I}$ over all asymptotically Euclidean four-geometries and matter fields with no asymptotic excitations which match the arguments of the wave function on $S$. The path integral over four-geometries will be equal to the sum of all the contributions which come from all asymptotically Euclidean four-metrics which satisfy the gauge-fixing condition (3.6). In order to sum each contribution only once, I shall divide the partial result of summing over all four-metrics which sastisfy (3.6) and that give a certain contribution by the corresponding volume in phase space, as required by the standard Faddeev-Popov techniques.

$$
\Psi\left[g_{i k}^{\prime}, \Phi^{\prime}\right]=\int \mathscr{D} N \mathscr{D} N^{i} \int_{C_{\mathrm{wh}}} \mathcal{D} g_{i k} \mathcal{D} \pi^{i k} \mathcal{D} \Phi \mathcal{D} \pi_{\Phi} \Delta_{\mathrm{FP}}\left(N, N^{i}\right) \delta(N-1) \delta\left(N^{i}\right) e^{-I\left[g_{i k}, N, N^{i}, \Phi\right]}
$$

where $\Delta_{\mathrm{FP}}\left(N, N^{i}\right)$ is the Faddeev-Popov determinant. It can be written in the form

$$
\Delta_{\mathrm{FP}}=\left[\int \mathcal{D} \epsilon^{\mu} \delta\left(\dot{\epsilon}^{\mu}-\kappa_{v^{\prime} \sigma^{\prime \prime}}^{\mu} N^{v^{\prime}} \epsilon^{\sigma^{\prime \prime}}\right)\right]^{-1},
$$

where one integrates over all gauge transformations that satisfy the conditions (3.4) and (3.5). The only history $\epsilon^{\mu}(x, \tau)$ that makes the argument of the $\delta$ function vanish and that satisfies (3.4) and (3.5) is $\epsilon^{\mu}(x, \tau)=0$ and, therefore,

$$
\Delta_{\mathrm{FP}}=1 .
$$

This was expected because there is only one history $N^{\mu}$ which satisfies the gauge-fixing condition, namely, $N=1$, $N^{i}=0$. Also, this can be interpreted as saying that the proper time between the three-surface $S$ and the asymptotically Euclidean region is fixed to an infinite value and, thus, one does not have to sum over all proper time separations between the two configurations which is always infinite. Therefore, it is straightforward to perform the path integration over the lapse $N$ and shift $N^{i}$ functions:

$$
\Psi\left[g_{i k}^{\prime},, \Phi^{\prime}\right]=\int_{C_{\mathrm{wh}}} \mathcal{D} g_{i k} \mathcal{D} \pi^{i k} \mathcal{D} \Phi \mathcal{D} \pi_{\Phi} e^{-I\left[g_{i k}, \Phi\right]},
$$

where

$$
\begin{aligned}
I\left[g_{i k}, \Phi\right]= & \int_{0}^{\infty} d \tau \int d^{3} x\left(\pi^{i k} \dot{g}_{i k}+\pi_{\Phi} \dot{\Phi}-\mathscr{H}\right) \\
& -\left.\int d^{3} x \pi^{i k} g_{i k}\right|_{\tau=\infty}
\end{aligned}
$$

and $C_{\mathrm{wh}}$ consists of all three-metrics and regular matter fields such that

$$
g_{i k}(x, 0)=g_{i k}^{\prime}(x), \quad \Phi(x, 0)=\Phi^{\prime}(x)
$$

and that satisfy suitable boundary conditions at infinity. These conditions are discussed in what follows.

In the gauge (3.6) the requirement that the spacetime must be asymptotically Euclidean is equivalent to the fixation of the asymptotic gravitational momentum which is given by

$$
\pi^{i k}(x, \infty)=-\frac{m_{P}^{2}}{8 \pi} \Omega^{1 / 2}(x) \Omega^{i k}(x),
$$

where $\Omega_{i k}$ is the metric of the unit three-sphere.

When the three-metric $g_{i k}^{\prime}$ and the matter field $\Phi^{\prime}$, which are the arguments of the wave function, approach the asymptotically Euclidean region, which is void of matter excitations, the wave function will be dominated by the saddle-point configuration that corresponds to this situation. This saddle point will exist only if the field configuration in the asymptotic region is homogeneous and the potential has a vanishing minimum at it. Otherwise, the saddle point will not be asymptotically Euclidean. Therefore, the wave function

$$
\Psi \sim \exp \left(\left.\int d^{3} x \pi^{i k} g_{i k}\right|_{\tau=0}\right)
$$

will be exponentially damped in the asymptotic region, as required. Thus, the boundary condition for the matter field at infinity will be

$$
\Phi(x, \infty)=\Phi_{0},
$$

where $\Phi_{0}$ is the asymptotic homogeneous field configuration at which the potential has a vanishing minimum. The boundary condition (4.7) means that 
there are no gravitational excitations at infinity, while (4.9) ensures that there are no asymptotic matter excitations either. These conditions together with (4.12) will pick the ground-state wave function for the wormhole. These results can be easily extended to the conformal case $\xi=1 / 6$.

$$
1-\frac{8 \pi}{m_{P}^{2}} \xi \Phi_{0}^{2}<0
$$

the effective gravitational constant in the asymptotic region, which from (2.2) can be seen to be

$$
G_{\mathrm{eff}}=G\left(1-\frac{8 \pi}{m_{P}^{2}} \xi \Phi_{0}^{2}\right]^{-1},
$$

is negative and gravity becomes repulsive. This problem has already been addressed by Halliwell and Laflamme [3] in a classical context. This fact is also reflected in the wormhole wave function: (4.8) will be exponentially enhanced rather than exponentially damped. It will not represent a wormhole anymore. Thus, the additional condition

$$
\Phi_{0}^{2}<\frac{m_{P}^{2}}{8 \pi} \xi^{-1}
$$

must be imposed (note that, for the minimal scalar field $\xi=0$, it is not a restriction). This requirement is not exclusive of wormholes. Rather, it is a natural condition that one has to impose in any reasonable theory: the effective gravitational constant must be positive in any region of spacetime in which observations are being made.

The wormhole wave function (4.4) is invariant under reparametrizations of the three-surface $S$. In fact, (3.5) was introduced just to keep this invariance during the gauge-fixing process. Also by construction, (4.4) is independent of the choice of the gauge-fixing condition. Finally, the wave function does not depend on the lapse or shift functions. Therefore, it satisfies, at least formally, the operator version of (2.15) (Ref. [20]):

$$
\mathscr{H} \Psi=0, \mathscr{H}_{i} \Psi=0 .
$$

The first equation is the Wheeler-DeWitt equation which reflects the fact that the path integral is invariant under time reparametrizations and the second one expresses the invariance of the wave function under changes of coordinates in the three-surface $S$.

\section{MINIMAL SCALAR FIELD}

Now, it is straightforward to construct the groundstate wave function for wormholes if one restricts to some minisuperspace models. I shall restrict the fourgeometries to have closed, homogeneous three-sections. The matter content will be a minimally coupled homogeneous massless scalar field.

\section{A. Friedmann-Robertson-Walker minisuperspace}

In this case, the spatial three-sections will also be isotropic and therefore, a single scalar factor will be sufficient. The FRW metric can be written

$$
d s^{2}=\sigma^{2}\left[\frac{d \tau^{2}}{2 q(\tau)}+2 q(\tau) d \Omega_{3}^{2}\right],
$$

where $\sigma^{2}=2 G / 3 \pi$. The three-metric and its conjugate momentum will be $g_{i k}=2 \sigma^{2} q \Omega_{i k}$ and $\pi^{i k}$ $=-(1 / 8 \pi G) \dot{q} \Omega^{1 / 2} \Omega^{i k}$, respectively. Note that a different convention for the lapse function from that in the preceding sections has been used. However, the result (4.4) does not depend on this choice that can be interpreted as coming from a different gauge fixation. Rescaling the scalar field $\Phi(\tau)=\sqrt{3 / 4 \pi G} \phi(\tau)$, the wave function (4.4) can be written

$$
\Psi\left(q^{\prime}, \phi^{\prime}\right)=\int_{C_{\mathrm{wh}}} \mathcal{D} q \mathcal{D} \pi_{q} \mathcal{D} \phi \mathcal{D} \pi_{\phi} e^{-I[q, \phi]},
$$

where

$$
\begin{aligned}
& I[q, \phi]=\int_{0}^{\infty} d \tau\left(\pi_{q} \dot{q}+\pi_{\phi} \dot{\phi}-H\right)-\left.q \pi_{q}\right|_{\tau=\infty}, \\
& H=\frac{1}{2}\left(-\pi_{q}^{2}+1\right)+\frac{1}{2} q^{-2} \pi_{\phi}^{2},
\end{aligned}
$$

$\pi_{\phi}$ is the momentum conjugate to $\phi$ and $C_{\mathrm{wh}}$ is the set of histories $\left(q, \pi_{q}, \phi, \pi_{\phi}\right)$ such that

$$
\begin{aligned}
& q(0)=q^{\prime}, \quad \phi(0)=\phi^{\prime}, \\
& \pi_{q}(\infty)=-1, \quad \phi(\infty)=\phi_{0} .
\end{aligned}
$$

Now, the potential is zero and, therefore, any asymptotic value of the field defines a ground state. There is an infinite number of ground states which are labeled by the asymptotic field value $\phi_{0}$.

With the change of variables [21]

$$
\begin{aligned}
& q=\left(x^{2}-t^{2}\right)^{1 / 2}, \quad \phi=\operatorname{arctanh} \frac{t}{x}, \\
& q \pi_{q}=x \pi_{x}+t \pi_{t}, \quad \pi_{\phi}=x \pi_{t}+t \pi_{x},
\end{aligned}
$$

the action takes the simple form

$$
I=\int_{0}^{\infty} d \tau\left(\pi_{x} \dot{x}+\pi_{t} \dot{t}-H\right)-\left.\left(x \pi_{x}+t \pi_{t}\right)\right|_{\tau=\infty},
$$

where

$$
H=\frac{1}{2}\left(-\pi_{x}^{2}+\pi_{t}^{2}+1\right) .
$$

The boundary conditions (5.5) and (5.6) transform into

$$
\begin{aligned}
& x(0)=q^{\prime} \cosh \phi^{\prime}, \quad t(0)=q^{\prime} \sinh \phi^{\prime}, \\
& \pi_{x}(\infty)=-\cosh \phi_{0}, \quad \pi_{t}(\infty)=\sinh \phi_{0},
\end{aligned}
$$

and the measure into $\mathcal{D} x \mathcal{D} t \mathscr{D} \pi_{x} \mathcal{D} \pi_{t}$, due to the fact that the Jacobian is equal to one.

To do the path integration

$$
\Psi_{\phi_{0}}\left(q^{\prime}, \phi^{\prime}\right)=\int \mathcal{D} x \mathcal{D} t \mathscr{D} \pi_{x} \mathcal{D} \pi_{t} e^{-I[x, t]},
$$

with the boundary conditions (5.11) and (5.12), it is convenient to shift the integration variables 


$$
\begin{aligned}
& x(\tau)=\bar{x}(\tau)+X(\tau), \quad t(\tau)=\bar{t}(\tau)+T(\tau) \\
& \pi_{x}(\tau)=\pi_{\bar{x}}+P_{X}(\tau), \pi_{t}(\tau)=\pi_{\bar{t}}(\tau)+P_{T}(\tau)
\end{aligned}
$$

where $\bar{x}, \pi_{\bar{x}}, \bar{t}$, and $\pi_{\bar{t}}$ correspond to the classical solution of the variational problem associated with (5.9) which satisfies the boundary conditions (5.11) and (5.12). Explicitly, this solution is

$$
\begin{aligned}
& \bar{x}(\tau)=\tau \cosh \phi_{0}+q^{\prime} \cosh \phi^{\prime}, \\
& \bar{t}(\tau)=\tau \sinh \phi_{0}+q^{\prime} \sinh \phi^{\prime} .
\end{aligned}
$$

The new variables $X$ and $T$ satisfy

$$
X(0)=T(0), \quad P_{X}(\infty)=P_{T}(\infty)=0 .
$$

Then, the action (5.9) splits into two parts

$$
I[x, t]=I_{0}[\bar{x}, \bar{t}]+I_{2}[X, T],
$$

where $I_{0}[\bar{x}, \bar{t}]$ is the action for the classical solution (5.16),

$$
I_{0}\left(q^{\prime}, \phi^{\prime} ; \phi_{0}\right)=q^{\prime} \cosh \left(\phi^{\prime}-\phi_{0}\right),
$$

and

$I_{2}[X, T]=\int_{0}^{\infty} d \tau\left[P_{X} \dot{X}+P_{T} \dot{T}-\frac{1}{2}\left(-P_{X}^{2}+P_{T}^{2}\right)\right]$.

The linear term $I_{1}[\bar{x}, \bar{t}, X, T]$ vanishes identically due to the fact that (5.16) is a saddle point of the action (5.9). The measure in the path integral (5.13) is directly changed into $\mathscr{D} X \mathscr{D P} P_{X} \mathcal{D} T \mathcal{D P}_{T} . I_{0}$ does not depend on $X$ or $T$ and then can be taken out of the integral. The remaining path integral becomes

$$
\int \mathcal{D} X \mathscr{D} T \exp \left[-\int_{0}^{\infty} d \tau \frac{1}{2}\left(-\dot{X}^{2}+\dot{T}^{2}\right)\right],
$$

which is independent of $q^{\prime}$ and $\phi^{\prime}$ and, therefore, it turns out to be a numerical factor [21]. Thus, up to numerical prefactors,

$$
\Psi_{\phi_{0}}(q, \phi)=e^{-q \cosh \left(\phi-\phi_{0}\right)},
$$

which had already been found as a solution of the Wheeler-DeWitt equation [15].

This wave function behaves in a regular way when the three-geometry degenerates. However, the linear combination

$$
\Psi_{k}(q, \phi)=\int_{-\infty}^{+\infty} d \phi_{0} e^{-i k \phi_{0}} \Psi_{\phi_{0}}(q, \phi)
$$

is an eigenfunction of the operator $\pi_{\phi}$ with eigenvalue $k$ and, therefore, has a nonzero flux through each threesurface. This wave function cannot close off with a fourgeometry and will oscillate an infinite number of times when the three-geometry collapses to zero. In fact, once the integral over $\phi_{0}$ is evaluated, the wave function (5.23) takes the form

$$
\Psi_{k}(q, \phi)=K_{i k}(q) e^{-i k \phi},
$$

where $K_{i k}(q)$ is a modified Bessel function of imaginary order. This wave function will oscillate for $q<|k|$, while for $q>|k|$, it will decrease exponentially. Thus, $|k|$ can be considered as the throat radius of the wormhole [13]. Since (5.23) is a Fourier transform, it suggests a kind of uncertainty relation between the asymptotic field and the wormhole throat radius. This could be expected beccause $k$ does not only represent the throat size but it is also the eigenvalue of the momentum conjugate to the scalar field and, therefore, $k$ and $\phi_{0}$ naturally satisfy an uncertainty relation [15].

\section{B. Kantowski-Sachs minisuperspace}

Another simple example for which the path integral that defines the ground-state wave function can be done explicitly is the minisuperspace model considered in [15]. It consists of a Kantowski-Sachs (KS) spacetime and a minimally coupled massless scalar field $\Phi(\tau)$ $=(4 \pi G)^{-1 / 2} \phi(\tau)$. The KS metric can be written in the form

$$
d s^{2}=\frac{G}{2 \pi}\left(e^{-2 \alpha} d \tau^{2}+e^{2 \alpha} d r^{2}+c^{2} e^{-2 \alpha} d \Omega_{2}^{2}\right),
$$

where $d \Omega_{2}^{2}$ is the metric in the unit two-sphere and $r$ is identified periodically. The Euclidean action for this minisuperspace is

$$
\widetilde{I}=\frac{1}{2} \int_{0}^{\infty} d \tau\left[-\dot{c}^{2}+c^{2}\left(\dot{\alpha}^{2}+\dot{\phi}^{2}\right)-1\right] .
$$

It is invariant under the action of the Euclidean group $E(2)$ on the $(\alpha, \phi)$ plane. Although the Wheeler-DeWitt equation will have also this symmetry, the path integral (and therefore, the solutions of the Wheeler-DeWitt equation) will not. This is because the boundary conditions that will be imposed are not invariant under $E(2)$. In fact, as is shown in [15], the different solutions of the Wheeler-DeWitt equation and, as we shall see, the path integral, i.e., the wormhole wave functions, will be labeled by an element of the group $E(2)$. The treatment is parallel to that in FRW spacetime. There, the Hamiltonian was invariant under the group of translations $E(1)$ in $\phi$. The boundary conditions (5.6) were not. The value of the field was fixed at infinity and this was the label of the wormhole wave function.

We consider spacetimes which are asymptotically $\mathbb{R}^{3} \times S^{1}$ in the KS minisuperspace model. In fact, these spacetimes are locally Euclidean and therefore, there are no gravitational excitations at infinity, although the topology is not $\mathbb{R}^{4}$. The mathematical condition that expresses this requirement is easily seen to be

$$
\dot{c}(\infty)=1, \quad \alpha(\infty)=\alpha_{0} .
$$

Note that, with this condition, we are fixing the $S^{1}$ radius to finite value $a_{0}=e^{\alpha_{0}}$. Also, we have to fix two more parameters: the asymptotic value of the field and the $\theta$ parameter that gives the polarization of the wave function in the plane $(\alpha, \phi)$, Therefore, we are fixing the three parameters of the group $E(2)$ in the asymptotic region:

$$
\alpha(\infty)=\alpha_{0}, \quad \phi(\infty)=\phi_{0}, \quad \theta=\theta_{0} .
$$


Once the three values in (5.28) have been chosen, define

$$
\varphi=\alpha \cos \theta_{0}+\phi \sin \theta_{0} .
$$

In terms of this new field and taking into account the surface term that appears because the spacetime is asymptotically $\mathbb{R}^{3} \times S^{1}$ the action is

$$
I=\frac{1}{2} \int_{0}^{\infty} d \tau\left(-\dot{c}^{2}+c^{2} \dot{\varphi}^{2}-1\right)+\left.c \dot{c}\right|_{\tau=\infty} .
$$

The same arguments that in the FRW minisuperspace led to (5.22), now, lead to

$$
\Psi_{\varphi_{0}}(c, \varphi)=e^{-c \cosh \left(\varphi-\varphi_{0}\right)} .
$$

If we undo the change (5.29) we get

$\Psi_{\alpha_{0}, \phi_{0}, \theta_{0}}(c, \phi, \alpha)=e^{-c \cosh \left[\left(\alpha-\alpha_{0}\right) \cos \theta_{0}+\left(\phi-\phi_{0}\right) \sin \theta_{0}\right]}$

which had been also previously obtained as a solution of the Wheeler-DeWitt equation [15]. This solution has the same structure as that in (5.22) and, mutatis mutandis, similar conclusions can be obtained.

\section{CONFORMAL SCALAR FIELD}

When a homogeneous scalar field $\Phi(\tau)$ $=\sqrt{3 / 4 \pi G} \phi(\tau)$ is conformally coupled to a FriedmannRobertson-Walker spacetime, the path integral which defines the wormhole wave function can also be done explicitly. The FRW metric can be written in the convenient form

$$
d s^{2}=\frac{2 G}{3 \pi} a^{2}\left(d \tau^{2}+d \Omega_{3}^{2}\right) .
$$

With the field redefinition

$$
\phi(\tau)=\chi(\tau) a^{-1}(\tau),
$$

the Euclidean action for this system becomes

$I=\frac{1}{2} \int_{0}^{\infty} d \tau\left(-\dot{a}^{2}-a^{2}+\dot{\chi}^{2}+\chi^{2}\right)+\left.\frac{1}{2} \frac{\dot{a}}{a}\left(a^{2}-\chi^{2}\right)\right|_{\tau=\infty}$.

The boundary conditions are in this csae

$$
\begin{aligned}
& a(0)=a^{\prime}, \quad \phi(0)=\phi^{\prime}=\frac{\chi^{\prime}}{a^{\prime}}, \\
& \frac{\dot{a}}{a}(\infty)=1, \quad \phi(\infty)=\frac{\chi}{a}(\infty)=\phi_{0} .
\end{aligned}
$$

The conditions at $\tau=0$ indicate which are the arguments of the wave function. The conditions (6.5) mean that the spacetime is asymptotically Euclidean and that the field $\phi$ takes the value $\phi_{0}$ at infinity. The wormhole wave function will be labeled by the asymptotic field value $\phi_{0}$ : being labeled by the asymptotic field value $\phi_{0}$ :

$$
\Psi_{\phi_{0}}\left(a^{\prime}, \chi^{\prime}\right)=\int_{C_{\mathrm{wh}}} \mathcal{D} a \mathcal{D} \chi e^{-I[a, \chi]},
$$

where $C_{\mathrm{wh}}$ is the class of histories that satisfy (6.4) and (6.5).

Let

$$
\begin{aligned}
& \bar{a}=a^{\prime} \cosh \tau+\frac{1+\phi_{0}^{2}}{1-\phi_{0}^{2}}\left[a^{\prime}-\frac{2 \phi_{0}}{1+\phi_{0}^{2}} \chi^{\prime}\right] \sinh \tau, \\
& \bar{\chi}=\chi^{\prime} \cosh \tau+\frac{1+\phi_{0}^{2}}{1-\phi_{0}^{2}}\left[\frac{2 \phi_{0}}{1+\phi_{0}^{2}} a^{\prime}-\chi^{\prime}\right] \sinh \tau
\end{aligned}
$$

be the solution of the classical equations of motion that satisfies (6.4) and (6.5). Then, as in the case of a minimal coupling and due to the fact that the action is also quadratic, under the shift of the integration variables

$$
a=\bar{a}+A, \quad \chi=\bar{\chi}+X,
$$

where the new variables $A$ and $X$ are such that

$$
A(0)=X(0)=0, \quad \frac{\dot{A}}{A}(\infty)=1, \quad \frac{X}{A}(\infty)=\phi_{0},
$$

the path integral (6.6) transforms into

$$
\Psi_{\phi_{0}}\left(a^{\prime}, \chi^{\prime}\right)=e^{-I_{0}\left(a^{\prime}, \chi^{\prime}\right)} \int \mathcal{D} A \mathcal{D} X e^{-I_{2}[A, X]},
$$

where $I_{2}[A, X]$ does not depend on $a^{\prime}$ or $\chi^{\prime}$ and

$I_{0}\left(a^{\prime}, \chi^{\prime}\right)=\frac{1}{2}\left(\frac{1+\phi_{0}^{2}}{1-\phi_{0}^{2}}\right)\left[a^{\prime 2}+\chi^{\prime 2}-\frac{4 \phi_{0}}{1+\phi_{0}^{2}} a^{\prime} \chi^{\prime}\right]$

is the action of the classical solution (6.7). Then, up to numerical factors,

$\Psi_{\phi_{0}}(a, \chi)=\exp \left[-\frac{1}{2}\left[\frac{1+\phi_{0}^{2}}{1-\phi_{0}^{2}}\right]\left[a^{2}+\chi^{2}-\frac{4 \phi_{0}}{1+\phi_{0}^{2}} a \chi\right)\right]$.

As was expected, this wave function is also a solution of the Wheeler-DeWitt equation. The condition

$$
\phi_{0}^{2}<1
$$

must be satisfied by the asymptotic field in order to have a positive effective gravitational constant in the asymptotic region, as discussed in Sec. IV. Then (6.12) will represent the wormhole wave function. Condition (6.13) will also allow us to write this wave function as a linear combination of those obtained in [2] and [13]:

$$
\Psi_{\phi_{0}}(a, \chi)=\sum_{n=0}^{\infty} \phi_{0}^{n}\left(1-\phi_{0}^{2}\right)^{1 / 2} \Psi_{n}(a, \chi),
$$

where

$\Psi_{n}(a, \chi)=\left(2^{n} n !\right)^{-1} H_{n}(a) e^{-a^{2} / 2} H_{n}(\chi) e^{-\chi^{2} / 2}$.

\section{SUMMARY AND CONCLUSIONS}

The purpose of this paper has been twofold. On the one hand, a path-integral formalism for wormholes has been developed in which the path integrations over the lapse and shift functions have been performed. The path integral over four-geometries has been thus reduced to a path integral over three-metrics. Also, the class of three-metrics and matter fields over which one has to integrate in order to get the wormhole wave functions has 
been precisely defined. On the other hand, this formalism has been applied to some minisuperspace models for which explicit wave functions were obtained.

I began in Sec. II with a review of the canonical formalism of general relativity. For the matter content, I considered a scalar field with a potential. Special attention was dedicated to writing a suitable action for asymptotically Euclidean spacetimes. Also in this section, was written the algebra of constraints, which was necessary to obtain the variation of this action under general gauge transformations. Then, it was shown that for any gauge transformation to leave the action invariant, one had to impose the condition that it mapped the three-surface $S$ onto itself. A new condition was introduced in order to keep the gauge freedom of the three-surface which was the argument of the wave function in the integration process. Also, an admissible gauge-fixing condition was given.

In Sec. IV, I performed the path integrations over the lapse and shift functions in an invariant way. Thus, the path integral that defined the wormhole wave function was reduced to a sum over three-metrics and matter fields for which suitable asymptotic boundary conditions were given. The requirement that the spacetime was asymptotically Euclidean was accomplished by fixing the asymptotic gravitational momentum, while the absence of asymptotic matter excitations required that the matter field had to approach asymptotically a homogeneous configuration at which the potential had a vanishing minimum. The necessity that the effective gravitational constant be positive in the asymptotic region also constrained the possible values of the asymptotic field for nonmininal couplings.

Section $\mathrm{V}$ was devoted to homogeneous minisuperspace models with a minimal massless scalar field. The path integration that defined the ground state was performed for both Friedmann-Robertson-Walker and KantowskiSachs minisuperspaces. The resultant wave functions had been previously obtained as solutions of the WheelerDeWitt equation and were written as Fourier transforms of those which represented wormhole throats with fixed radii. The throat radius and the asymptotic field satisfied a kind of uncertainty relation.

The case of a massless scalar field conformally coupled to a homogeneous and isotropic spacetime was studied in Sec. VI. For the physical region of the spectrum, i.e., for those asymptotic field values which made the effective gravitational constant in the asymptotic region positive, I obtained a wave function which was written as a linear combination of products of harmonic-oscillator wave functions.

\section{ACKNOWLEDGMENTS}

I am very grateful to Pedro González-Díaz for many enlightening discussions and encouraging comments. Also, I wish to thank Guillermo Mena Marugán for valuable explanations and Jonathan Halliwell for useful conversations and for introducing me to some references relevant to this work.
[1] S. B. Giddings and A. Strominger, Nucl. Phys. B306, 890 (1988).

[2] S. W. Hawking, Phys. Rev. D 37, 904 (1988).

[3] J. J. Halliwell and R. Laflamme, Class. Quantum Grav. 6, 1839 (1989).

[4] A. Hosoya and W. Ogura, Phys. Lett. B 225, 117 (1989).

[5] B. J. Keay and R. Laflamme, Phys. Rev. D 40, 2118 (1989).

[6] P. F. González-Díaz, Phys. Lett. B 247, 251 (1990).

[7] G. A. Mena Marugán, Class. Quantum Grav. 8, 935 (1991).

[8] S. Coleman, Nucl. Phys. B310, 643 (1988).

[9] S. B. Giddings and A. Strominger, Nucl. Phys. B307, 854 (1988).

[10] S. W. Hawking, Nucl. Phys. B335, 155 (1990).

[11] S. Bochner, Bull. Am. Math. Soc. 52, 776 (1946).

[12] J. Cheeger and D. Gromoll, Ann. Math. 96, 413 (1972).

[13] S. W. Hawking and D. B. Page, Phys. Rev. D 42, 2655
(1990).

[14] P. F. González-Díaz, Nucl. Phys. B351, 767 (1991).

[15] L. M. Campbell and L. J. Garay, Phys. Lett. B 254, 49 (1991).

[15a] G. A. Mena Marugán (private comunication).

[16] S. W. Hawking, in General Relativity: An Einstein Centenary Survey, edited by S. W. Hawking and W. Israel (Cambridge University Press, Cambridge, England, 1979).

[17] C. Teitelboim, Phys. Rev. D 28, 297 (1983).

[18] K. Kuchař, in Quantum Gravity 2: A Second Oxford Symposium, edited by C. J. Isham, R. Penrose, and D. W. Sciama (Clarendon, Oxford, 1981).

[19] C. Teitelboim, Phys. Rev. D 25, 3159 (1982).

[20] J. J. Halliwell and J. B. Hartle, Phys. Rev. D 43, 1170 (1991).

[21] L. J. Garay, J. J. Halliwell, and G. A. Mena Marugán, Phys. Rev. D 43, 2572 (1991). 